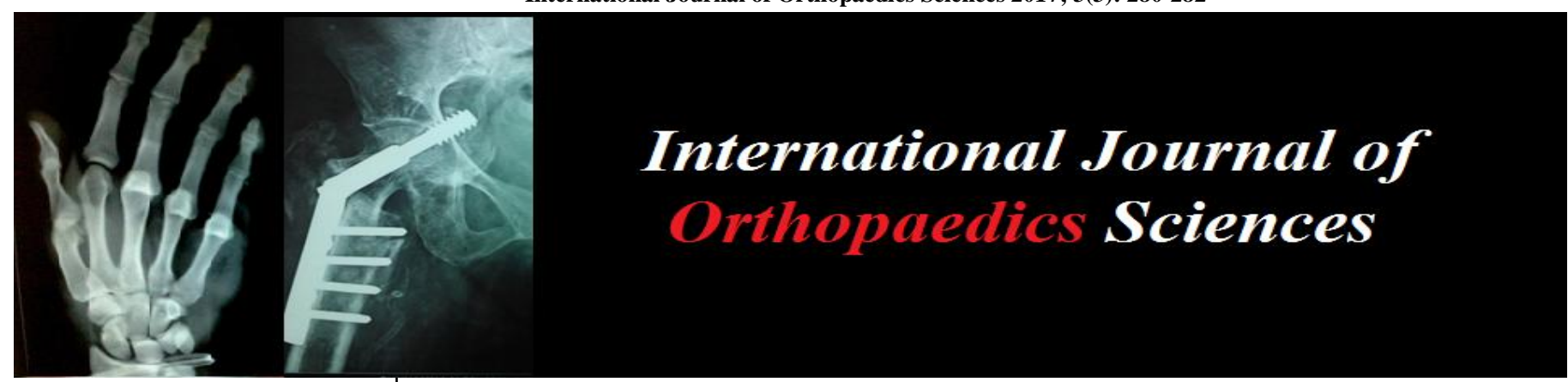

ISSN: $2395-1958$

IJOS 2017; 3(3): 280-282

(C) 2017 IJOS

www.orthopaper.com

Received: 11-05-2017

Accepted: 12-06-2017

Dr. Darsh Goyal

Orthocare Centre, Janakpuri

West, New Delhi, India

Dr. Radhey Sham Garg

Professor, Department of

Orthopaedics, GGS Medical

College and Hospital, Faridkot,

Punjab, India

Dr Mohammed Sagy

Resident GGS Medical College,

Faridkot, Punjab, India

Dr. Gursagar Deep Singh Resident, GGS Medical College,

Faridkot, Punjab, India

\section{Role of platelet rich plasma in osteoarthritis knee}

\section{Dr. Darsh Goyal, Dr. Radhey Sham Garg, Dr. Mohammed Sagy and Dr. Gursagar Deep Singh}

DOI: http://dx.doi.org/10.22271/ortho.2017.v3.i3e.51

\section{Abstract}

Worldwide osteoarthritis is the most common joint disorder. It results from mechanical and biological events that destabilize the normal processes of degradation and synthesis of articular cartilage chondrocytes, extracellular matrix and subchondral bone. These changes include increased water content, decreased proteoglycan content and altered collagen matrix, leading to the degeneration of articular cartilage. There is trend on rise to use platelet rich plasma to promote healing of the degenerated cartilage. The aim of the study was to evaluate whether the newer method i.e platelet rich plasma has any significant advantage. The study included a total of 50 cases with individuals ageing greater than 40 years. Patients without evidence of degenerative arthritis and with KL grade 3 and grade 4 were excluded from the study. At 1 month only 14\% cases showed excellent, $68 \%$ cases showed and. $18 \%$ cases showed fair results. At 3 months follow up 16\% cases showed excellent, $82 \%$ cases showed good and fair results were seen in $2 \%$ cases. No case had poor results. At 6 months follow up $16 \%$ cases showed excellent, $86 \%$ cases showed good and 6\% cases showed fair results and no case had poor results. Platlet rich plasma is an excellent method of treatment in early osteoarthritis of knee.

Keywords: Osteoarthritis, functional outcome, knee, degereration

\section{Introduction}

Osteoarthritis is a condition with a long history, and has been identified in both ancient and modern animals and humans. Until the 1980s; OA was considered a primarily degenerative disorder and a natural occurrence of "wear-and-tear" on joints as a result of aging. Recent research evidence is changing this view, as knowledge increases regarding the pathogenesis and natural history of OA, particularly the metabolically active role of the disease and the process of remodeling and repair of damaged tissue. Current thinking is that it may be possible to arrest the progress of, and potentially even reverse, the disease ${ }^{[1]}$. It results from mechanical and biological events that destabilize the normal processes of degradation and synthesis of articular cartilage chondrocytes, extracellular matrix and subchondral bone. These changes include increased water content, decreased proteoglycan content and altered collagen matrix, leading to the degeneration of articular cartilage.

The present study focuses on the treatment of osteoarthritis in knee with platelet rich plasma.

\section{Material and Methods}

This prospective study was performed at a teaching hospital in India. The study consisted of a total of 50 cases. A case of either sex $\geq 40$ years old with osteoarthritis knee KellgrenLawrence Grade 1 and 2 were included into the study. An informed written consent of the patient was obtained before inclusion in the study. The ethical clearance from the ethics committee of the institute was obtained before the start of study.

In Group B, 50 patients were treated with intraarticular injection of autogenous growth factor rich plasma. Patients were discharged immediately after the procedure in both the groups. Following discharge from the hospital, the participants were followed up on a regular basis with clinical examinations and functional evaluations for pain relief as per VAS scale. All examinations were taken at 1 month, 3 months and 6 months.

\section{Preparation of Growth factor rich plasma}

On the day of the surgery, the patient was taken to the Blood Bank, GND Hospital, Amritsar. A three chambered blood bag was taken, and $48 \mathrm{ml}$ of CPD (citrate phosphate dextrose) was 
removed and discarded, leaving just $15 \mathrm{ml}$ of CPD in the bag. $100 \mathrm{ml}$ of patient's whole blood was drawn by a clean, single venipuncture, into the 1 st chamber of the blood bag. The bag was kept at room temperature (20-22 degrees C) before preparing platelet concentrate for not more than 6 hours. The bag was kept in the bucket of refrigerated centrifuge (Heraeus Cryofuge 6000i) and balanced accurately, and centrifuged at $2000 \mathrm{rpm}$ at 22 degrees $\mathrm{C}$ for 10 minutes. This separated the whole blood into red blood cell concentrate at the bottom and plasma above. $4 / 5$ th of the plasma was separated into the 2 nd satellite bag, double sealing the tubing between the primary bag and the satellite bag. The primary bag with RBC concentrate was separated and kept aside. The remaining 2 satellite bags were again centrifuged at $4000 \mathrm{rpm}$ at 22 degrees $\mathrm{C}$ for 10 minutes after balancing accurately. The plasma got separate into an upper layer of platelet poor plasma (PPP) and platelet concentrate (GFRP) below. The PPP layer was expressed into the 2nd satellite bag, double sealed, separated and kept aside. The GFRP (platelet concentrate) extract in the 1 st satellite bag was approximately $12 \mathrm{ml}$. This bag was sent to the OT immediately, where it was kept at room temperature before use.

\section{GFRP injection procedure}

GFRP was removed from the blood bag using aseptic technique, and was put in a sterile container. Affected knee was exposed and after proper cleaning \& draping knee joint was approached through superolateral site using $18 \mathrm{G}$ disposable steel spinal needle. GFRP was injected in the knee joint with the help of a $20 \mathrm{cc}$ syringe. Needle was removed \& aseptic dressing done. Patient was instructed to bend and extend the knee to allow equal distribution of GFRP throughout the joint. Patient was discharged with instruction to gradual resumption of normal activities.

\section{Evaluation of results}

Results were evaluated according to Visual Analogue Scale (VAS) (figure 1). Before the surgical procedure the pain was considered at $100 \mathrm{~mm}$ in all patients and at every follow up; patient was asked to mark a point on the line to explain how much of pain relief he/she is having.

Results evaluation as per VAS Scale improvement

$\begin{array}{lll}\text { Excellent } & >80 \% & \text { Improvement } \\ \text { Good } & 60-80 \% & \text { Improvement } \\ \text { Fair } & 40-60 \% & \text { Improvement } \\ \text { Poor } & <40 \% & \text { Improvement }\end{array}$

The evaluation of the results of clinical outcome of autogenous growth factor rich plasma (GFRP) injection in osteoarthritis knee was done.

\section{Results}

The mean age of the patients was 60.5 years. In our study females outnumbered males in presenting with pain due to knee OA. Female to male ratio was 7:3. Ratio of side of limb involvement was left to right 9:11. As per there is no specific side predilection for knee osteoarthritis. The targeted study population with Kellgren-Lawrence Grade 1 and 2 were recruited in this study. Ratio of patients with Grade 1 to 2 was $2: 3$. VAS score was considered as 100 in all the patients at the start of the study. At 1 month, 3 months and 6 months interval patients were asked to mark a point for the level of pain on a straight unmarked line $100 \mathrm{~mm}$ long. Once the marking was done by a patient, it was measured with a measuring scale to know VAS score. Lesser the VAS score better was the pain relief. At 1 month VAS score ranged from 17 to $35 \mathrm{~mm}$ at 1 month and mean score of 26.7 and standard deviation of 8.60766. At 3 months VAS score ranged from 17 to $31 \mathrm{~mm}$ and mean score of 24.9 and standard deviation of 6.96126. At 6 months VAS score ranged from 18 to $28 \mathrm{~mm}$ and mean score of 23.6 and standard deviation of 5.62792. (table I). At 1 month only $14 \%$ cases showed excellent, $68 \%$ cases showed and. $18 \%$ cases showed fair results. At 3 months follow up $16 \%$ cases showed excellent, $82 \%$ cases showed good and fair results were seen in $2 \%$ cases. No case had poor results. At 6 months follow up $16 \%$ cases showed excellent, $86 \%$ cases showed good and $6 \%$ cases showed fair results and no case had poor results.

There was aggravation of pain for about 24 hours in three cases. None of the cases experienced any deep or superficial infection.

Table I: Visual Analogue scale score at presentation and follow up

\begin{tabular}{|c|c|c|c|c|}
\hline & Baseline & 1 month & 3 months & 6 months \\
\hline Valid & 50 & 50 & 50 & 50 \\
\hline Mean & 100.0000 & 26.7000 & 24.9000 & 23.6000 \\
\hline Std. Error of Mean & .00000 & 1.21731 & .98447 & 79591 \\
\hline Std. Deviation & .00000 & 8.60766 & 6.96126 & 5.62792 \\
\hline Range & .00 & 25.00 & 25.00 & 25.00 \\
\hline Minimum & 100.00 & 15.00 & 15.00 & 15.00 \\
\hline Maximum & 100.00 & 40.00 & 40.00 & 40.00 \\
\hline
\end{tabular}

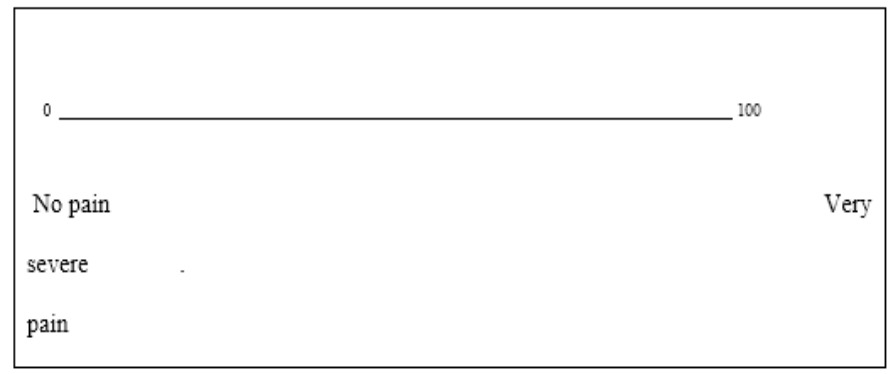

Fig 1: Visual Analogue Scale

\section{Discussion}

Osteoarthritis (OA) is the most common joint disease in the world, with an age-associated increase in both incidence and prevalence. OA is one of the leading causes of disability in the world, with more than $10 \%$ of the elderly population having symptomatic disease. In osteoarthritis of the knees, the microscopic degenerative debris are persistent cause of chemical and mechanical irritation during walking and knee movement, resulting in aggravation of pain. By mediating chondroprotective action PRP therapies can delay joint deterioration by interfering with the early catabolic and inflammatory events and by promoting anabolic responses subsequently reducing OA pain.

In the present study maximum patients were in the age group of 5th and 6th decade. The youngest patient in the study had an age of 42 years and the oldest had an age of 80 years with mean age of 60.5 years. Similar trend was seen in a series of Lawrence RC et al. ${ }^{[2]}$ while estimating the prevalence of arthritis and selected musculoskeletal disorders. The incidence increases with age, and by age 65 approximately $80 \%$ have radiographic evidence of OA. The peak performance of an individual is considered to be around age of 30 years and after that physical decline starts gradually. Around the age of 50 years, the decline becomes fast and as such the degenerative changes start appearing 
symptomatically. In females because of hormonal changes due to menopause the degenerative changes start appearing early.

In our study of 100 cases, there were 71 females and 29 male patients with female to male ratio of approximately 7:3. Similar trend was observed in the series of Woolf et al. ${ }^{[3]}$ in which male to female ratio was 7:3 and Gabriel Frías et al. ${ }^{[4]}$ in their study on 299 patients with male female ratio was 7.8: 2.2. Female predominance is attributed to the hormonal changes due to menopause resulting in early onset of degeneration and the lesser bone mass. In India the scenario is worsened for females because of social habits of squatting during worshipping, cleaning and mopping of household etc.

Kon $\mathrm{E}$ et al. ${ }^{[5]}$ in a prospective study on one hundred consecutive patients, affected by chronic degenerative condition of the knee, treated with PRP intra-articular injections were clinically evaluated before and at 6 and 12 months follow-up. IKDC, objective and subjective, and EQ VAS were used for clinical evaluation. A statistically significant improvement of all clinical scores was obtained from the basal evaluation to 6 and12 months follow-up ( $\mathrm{P}<$ 0.0005). The results remained stable upto 6 months follow up. Similar sustained pain relief was observed with PRPP injection in our study, indicating that the treatment with PRP injections is safe and has the potential to reduce pain and improve knee function and quality of life. Initial pain relief is because of viscosupplementation effect of PRPP and as the time passes the chondroprotective and chondroregeneration effect starts and hence a pain relief at later period is better. In our study pain relief was better at later stages.

No major complication such as deep infection, muscle atrophy, deep vein thrombosis, fever, hematoma, tissue hypertrophy, adhesion formation, or other major adverse events occurred among study subjects. Similar studies by Sampson S et al. ${ }^{[6]}$, Filardo G et al. ${ }^{[7]}$, Kon E et al. ${ }^{[5]}$, Sun Y et al. ${ }^{[8]}$ and Giannini $\mathrm{S}$ et al. ${ }^{[9]}$ showed no major complication or adverse effects.

In our study, three cases in GFRP group showed temporary worsening of pain for about 24 hours which resolved spontaneously. This is attributed to the sudden increase in the volume of intracapsular contents leading to capsular stretch and hence pain. Once some absorption occurs the stretch pain settles down. Spakova' T et al. ${ }^{[10]}$ in their study documented temporary mild worsening of pain in the knee joint after application of PRP in six cases out of 60, which was spontaneously resolved after 2 days.

\section{Conclusion}

Majority of the patients seek medical Intervention for their pain relief for osteoarthritis knee in the 5th decade of their life and when the problem is slightly advanced. Females suffer more than the males, the observations being attributed to the post-menopausal hormonal changes in the females. PRPP injection result in chondroprotective and chondroregenerative potential in knee joint cartilage and hence result in pain relief. It takes some time to induce the action and hence the onset of pain relief is slow but long lasting. We conclude that PRRP injection are excellent measures to relieve pain in early stages of O.A. knee (grade 1 and 2, as per Kellgren-Lawrence grading).

\section{Acknowledgements: None Declared}

\section{Reference}

1. Bland JH. The reversibility of osteoarthritis: a review.
Am J Med. 1983; 74(6A):16-26.

2. Lawrence RC, Helmick CG, Arnett FC, Deyo RA. Estimates of the prevalence of arthritis and selected musculoskeletal disorders in the United States. Arthritis Rheum. 1998; 41(5):778-99.

3. Woolf AD, Pfleger B. Burden of major musculoskeletal conditions. Bull World Health Organ. 2003; 81(9):64656.

4. Frías G, Caracuel MA, Escudero A, Rumbao J, PérezGujo V. Assessment of the efficacy of joint lavage versus joint lavage plus corticoids in patients with osteoarthritis of the knee. Curr Med Res Opin. 2004; 20(6):861-7.

5. Kon E, Buda R, Filardo G, Di Martino A, Timoncini A, Cenacchi A et al.. Platelet-rich plasma: intra-articular knee injections produced favorable results on degenerative cartilage lesions. Knee Surg Sports Traumatol Arthrosc. 2010; 18(4):472-9.

6. Sampson S, Reed M, Silvers H, Meng M, Mandelbaum B. Injection of platelet-rich plasma in patients with primary and secondary knee osteoarthritis: a pilot study. Am J Phys Med Rehabil. 2010; 89(12):961-9.

7. Filardo G, Kon E, Pereira Ruiz MT, Vaccaro F. Plateletrich plasma intra-articular injections for cartilage degeneration and osteoarthritis: single- versus doublespinning approach. Knee Surg Sports Traumatol Arthrosc. 2012; 20(10):2078-87.

8. Sun Y, Feng Y, Zhang CQ. The regenerative effect of platelet-rich plasma on healing in large osteochondral defects. Int Orthop. 2010; 4:589-97.

9. Giannini S, Vannini F, Timoncini A, Ghermandi R, Ruffilli A. The treatment of severe chondropaties of the knee with autologous platelet rich plasma Injections: Preliminary Results. Osteoarthritis and Cartilage. 2008; 16:126-7.

10. Spakova' T, Rosocha J, Lacko M, Harvanova' D, Gharaibeh A. Treatment of knee joint osteoarthritis with autologous platelet-rich plasma in comparison with hyaluronic acid. Am J Phys Med Rehabil. 2012; 91(5):411-7. 